\title{
Isotopic Analysis of Irradiated Ceramic Fuel for Burnup and Microchemical Assessment Using Atom Probe Tomography.
}

\author{
Mukesh Bachhav ${ }^{1}$, Joshua Kane ${ }^{2}$, Fei Teng ${ }^{2}$, Fabiola Cappia ${ }^{2}$ and Lingfeng $\mathrm{He}^{3}$ \\ ${ }^{1}$ Idaho National Laboratory, United States, ${ }^{2}$ INL, United States, ${ }^{3}$ Idaho National Laboratory, Idaho Falls, \\ Idaho, United States
}

Ceramic nuclear fuels, such as $\mathrm{UO}_{2}$ and mixed oxide (MOX), are the primary fuels used in the current commercial light water reactors and are also fuel candidates in advanced reactors. Oxide fuels are known for their thermo-mechanical and adequate physico-chemical stability under harsh operating condition of nuclear reactors and are hence the preferred fuel of choice. The energy supplied by uranium (U) based fuel is determined by consumption of U-235 isotope. This burnup of fuel leads to the large number of fission products (FP) generated during burnup which can impact the chemical and mechanical properties of the fuel. Precise knowledge on $U$ isotope quantification is desired for burnup assessment and its correlation to distribution of different fission products and its chemical states.

Atom probe microscopy is a unique and highly accurate technique for chemical quantification from materials based on mass spectrometric analysis. Accurate quantitative analyses using atom probe tomography (APT) is challenging for oxide samples with poor conductivity due to significant peak overlap within the mass spectrum. This problem gets more severe when peaks show thermal tail (shown in figure 1) from delayed field evaporation of ions and effectively overlapping preceding signal [1]. It is not possible to apply a single model peak approach universally to all peaks in given mass spectrum or between data sets of sample with poor conductivity.

In this work, we are developing an automated multi- asymmetric peak fitting approach to better resolve isotopic abundances in nuclear fuels and materials [2]. The process first determines an approximate peak fit serially for consecutive overlapping peaks subtracting out the contribution of prior peaks (figure 2). Once approximate parameters are determined, the parameters for each peak are optimized simultaneously. We utilize a constrained optimization method to take advantage of a priori information regarding general peak shape and character. The optimization algorithm utilizes an interior point algorithm capable of significant speed up via parallel processing. 


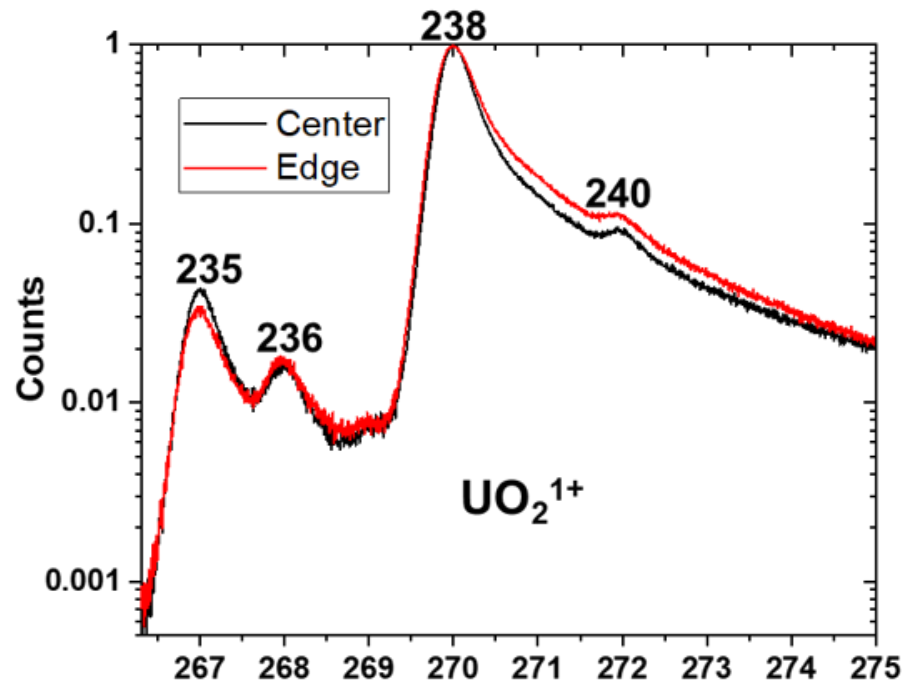

Figure 1. Mass spectrum of spent UO2 fuel for UO21+ ions showing different isotopes.

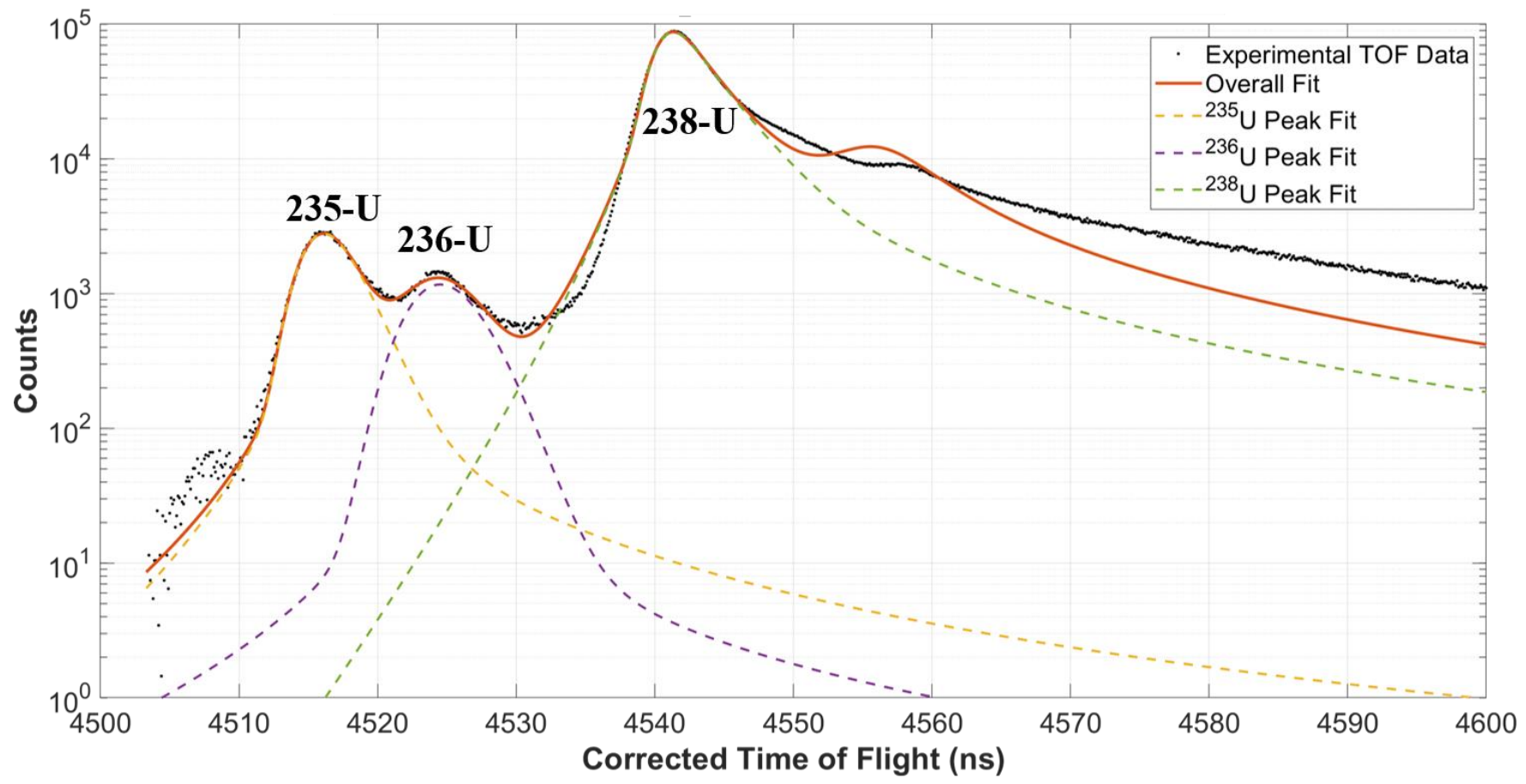

Figure 2. Fitting of time-of-flight mass spectrum of spent UO2 using Asymmetric Psuedo-Voigt probability distribution function for isotopic analysis.

References

[1] Frederick Meisenkothen, Daniel V. Samarov, Irina Kalisha, Eric B. Steel, Ultramicroscopy 216 (2020) 113018

[2] Aaron L. Stancik, Eric B. Brauns, Vibrational Spectroscopy 47 (2008) 66-69, 2008 